\title{
Epilepsy: A Spectrum Disorder
}

\author{
Joseph I. Sirven \\ Department of Neurology, Mayo Clinic in Arizona, Phoenix, Arizona 85054 \\ Correspondence: sirven.joseph@mayo.edu
}

Epilepsy, a disorder of unprovoked seizures is a multifaceted disease affecting individuals of all ages with a particular predilection for the very young and old. In addition to seizures, many patients often report cognitive and psychiatric problems associated with both the seizures themselves and its therapy. Epilepsy has numerous etiologies both idiopathic and acquired with a wide range of therapeutic responses. Despite numerous treatments available to control repetitive seizures including medications, diets, immunotherapy, surgery, and neuromodulatory devices, a large percentage of patients continue to suffer the consequences of uncontrolled seizures, which include psychosocial stigma and death.

Epilepsy is an ancient disorder and has been Edescribed historically from the times of the ancient Babylonians to the modern day. Although epilepsy is characterized by seizures that are unpredictable in frequency, epilepsy is a common neurological disorder that affects people of all ages. Indeed, epilepsy has a bimodal onset that occurs most often in childhood and older adulthood (Institute of Medicine 2012). However, it is also a spectrum of disorders with a range of severities, widely differing seizure types and causes, and varying impacts on individuals and their families. Beyond actually living with epilepsy and its seizures and coexisting health conditions, the challenges that face millions of people living with epilepsy include having access to high-quality healthcare, learning about and coordinating healthcare, medication, vocational, independent living, and other community services, and dealing with stigma and common public misunderstandings. As such, epilepsy imposes an immense burden on individuals, families, and society as a whole.
The Institute of Medicine report, "Epilepsy Across the Spectrum" summarized some important statistics regarding epilepsy (Institute of Medicine 2012). Epilepsy involves 2.2 million people in the United States and $>65$ million people worldwide (Hirtz et al. 2007). One hundred and fifty thousand new cases of epilepsy are diagnosed in the United States annually (Institute of Medicine 2012). One in 26 people in the United States will develop epilepsy at some point in their lifetime (Institute of Medicine 2012). Children and older adults are the fastest-growing segments of the population with new cases of epilepsy. The risk of death increases for people with epilepsy with an estimated 10 years of life lost for people whose epilepsy has a known cause, and 2 years lost for people with epilepsy from an unknown cause (Institute of Medicine 2012). The number of people with epilepsy who die from sudden unexpected death in epilepsy (SUDEP) varies from one of every 10,000 newly diagnosed to nine of every 1000 candidates for epilepsy surgery (Institute of Medicine 2012).

Editors: Gregory L. Holmes and Jeffrey L. Noebels

Additional Perspectives on Epilepsy: The Biology of a Spectrum Disorder available at www.perspectivesinmedicine.org

Copyright (C) 2015 Cold Spring Harbor Laboratory Press; all rights reserved; doi: 10.1101/cshperspect.a022848

Cite this article as Cold Spring Harb Perspect Med 2015;5:a022848 


\section{J.I. Sirven}

The direct annual medical care cost of epilepsy in the United States totals $\$ 9.6$ billion, and this does not consider community service costs or indirect costs from losses in quality of life and productivity (Begley et al. 2000). These indirect costs are estimated to constitute the majority of the cost burden of epilepsy.

\section{DEFINITION}

To understand seizures and epilepsy, one must fully grasp the official terminology and nomenclature used to describe seizures and epilepsy. According to the International League Against Epilepsy (ILAE), the main governing body responsible for terminology and nomenclature as it pertains to seizures and epilepsy, a seizure is defined as an abnormal electrical perturbation resulting from a network of neurons (Berg et al. 2010). In the year 2014, an ILAE task force revised the definition of epilepsy (Fisher et al. $2005,2014)$. A person is considered to have epilepsy if they meet any of the following conditions:

1. At least two unprovoked or reflex seizures occurring $>24$ hours apart.

2. One unprovoked or reflex seizure and a probability of further seizures similar to the general recurrence risk of at least $60 \%$ after two unprovoked seizures occurring over the next 10 years.

3. A diagnosis of an epilepsy syndrome.

As such, it is possible that someone can have the diagnosis of epilepsy after having one seizure depending on the etiology and the electroclinical syndrome.

Epilepsy is considered to be resolved for individuals who have an age-dependent epilepsy syndrome but are now past the applicable age or those who have remained seizure free for the last 10 years with no seizure medicines for the last 5 years. According to this new definition, epilepsy is now called a disease rather than a disorder, similar to other heterogeneous disorders like cancer and heart disease, which are also termed diseases to convey the seriousness of the condition to lay audiences.

\section{CLASSIFICATION OF SEIZURES AND EPILEPSY}

In all classification systems, the distinction between seizures primarily relies on whether the inception of these events in the cerebral cortex is of a focal or generalized origin. Therefore, all classification systems begin with a division of seizures between focal and generalized seizures. This is important because the choice of medical and surgical interventions will be dependent on the appropriate classification. The subtypes for generalized and focal seizures are displayed in Tables 1 and 2 .

Focal seizures originate at some point within networks limited to one hemisphere. Focal seizures may originate within subcortical structures and they may be classified as focal without impairment of consciousness (clonic, autonomic, and hemiconvulsive), focal or subjective sensory or psychic phenomenon (aura specific), focal dyscognitive with impairment of consciousness, and focal evolving to a bilateral convulsive seizure. The term simple partial, complex partial, and partial seizure with secondary generalization has been embedded in the epilepsy lexicon for decades. There has been considerable resistance of letting go of these terms. However, simple means without alteration of consciousness, and complex implies altered awareness. Complex partial has been replaced by the term focal dyscognitive describing seizures with disturbed cognition as the prominent feature. The term secondary generalized seizure is replaced by focal seizure evolving to a bilateral convulsive seizure.

Table 1. Focal seizures-three subtypes

\begin{tabular}{lc}
\hline Types & Other names \\
\hline $\begin{array}{l}\text { Focal seizures without } \\
\text { impairment of consciousness }\end{array}$ & Simple partial \\
Autonomic & \\
Clonic & \\
$\begin{array}{l}\text { Hemiconvulsive } \\
\text { Subjective sensory/psychic }\end{array}$ \\
$\begin{array}{l}\text { Focal dyscognitive seizures with } \\
\text { impairment of consciousness }\end{array}$ & Complex partial \\
$\begin{array}{l}\text { Focal seizure evolving to a bilateral } \\
\text { convulsive seizure }\end{array}$ & $\begin{array}{c}\text { Secondary } \\
\text { generalized }\end{array}$ \\
\hline
\end{tabular}


Table 2. Generalized seizures_-six subtypes

Primary tonic-clonic

Absence

Typical

Atypical

Absence with special features

Myoclonic absence

Eyelid myoclonia

Myoclonic

Tonic

Clonic

Atonic

Generalized seizures imply a bilateral hemispheric onset and they are divided among six categories. These include tonic-clonic, absence with five subdivisions (typical, atypical, absence with special features, myoclonic absence, and eyelid myoclonia), myoclonic with three subtypes (myoclonic, myoclonic atonic, and myoclonic-tonic), tonic, clonic, and atonic.

Neonatal seizures are no longer regarded as a separate entity. Seizures in neonates can be classified within this new scheme. Epileptic spasms are the acceptable term for infantile spasms because they may continue or begin after the first year of life. Because there is insufficient knowledge to classify these seizures as focal, generalized, or both, they have been placed in their own group.

\section{THE EPIDEMIOLOGY OF EPILEPSY}

The common standard measures of frequency of epilepsy in a population are incidence rate and prevalence rate. The incidence studies of epilepsies show that the age-adjusted incidence of epilepsy ranges from 16 per 100,000 person years to 111 per 100,000 person years depending on geographical location (Lavados et al. 1992; Benn et al. 2008). The highest age-adjusted incidence in the world is in rural Chile with 111 cases per 100,000 (Lavados et al. 1992). Most studies show a range from 26 per 100,000 person years (in Norway) to 47 per 100,000 person years (in England) (Brewis 1966; De Graaf 1974).

Point prevalence is a proportion of individuals in the population who are affected by health conditions at a single point and time. A plethora of studies have shown that the typical point prevalence for epilepsy is between four and 10 per 1000 persons (Sander 2003). Most large-scale studies of population and resource-poor countries have reported prevalence rates for active epilepsy as high as 6-10 per 1000 (Placencia et al. 1992; Aziz et al. 1994; Sander and Shorvon 1996; Bell and Sander 2001). Many of these studies have, however, reported different rates for urban and rural areas, usually with higher rates in the latter. The differences seem likely to be artifactual or attributable to differential case ascertainment between regions, as many of these studies used identical method study designs and diagnostic confirmation. Real differences in prevalence may be related to the presence of endemic conditions, such as neurocysticercosis or malaria, the medical infrastructure in place, including availability of preventive regional health programs, and accessible local medical care (Institute of Medicine 2012).

Lifetime prevalence is the risk of having a nonfebrile epileptic seizure at some point in an average lifetime. In both industrialized and resource poor countries, up to $5 \%$ of a population will experience a nonfebrile seizure at some point in their lives (Bell and Sander 2001; Sander 2003). From the difference between lifetime prevalence and the point prevalence of active epilepsy, it is apparent that in most patients who develop epilepsy, the condition remits.

Epilepsy, however, is associated with increased mortality, particularly, but not exclusively, in symptomatic cases. Different measures are used to estimate mortality depending on study design and available information. Mortality is best expressed as a standardized mortality ratio, which is the ratio of observed deaths (number of deaths in an epilepsy population) to that expected based on the age- and sex-specific mortality rates as a reference population in a given time. The proportional mortality ratio is the proportion of death owing to a particular cause in a cohort of patients in a given period and can be used to compare the relative contribution of various causes to the overall mortality in a population. 
In large cohort studies of patients $>15$ years of age, the standardized mortality ratio ranged from 2.1 to 5.1 (Nashef et al. 1995; Nilsson et al. 1997; Shackleton et al. 1999; Lindsten et al. 2000; Lhatoo et al. 2001). The majority of deaths in people whose seizures started in childhood occur in adulthood. Prospective studies in which children with large sample size were followed for 15 to $>30$ years reported high, standardized mortality rates (Harvey et al. 1993; Kurtz et al. 1998; Sillanpää et al. 1998; Camfield et al. 2002).

Death in people with epilepsy can be classified into three groups: epilepsy-related deaths, deaths related to the underlying cause of the epilepsy, and deaths that are unrelated to the epilepsy or underlying etiology. The proportional mortality ratio (PMR) for epilepsy-related conditions, range from $1 \%$ to $45 \%$ (Zielinski 1974; Hauser et al. 1980; Annegers et al. 1984; Cockerell et al. 1994; Loiseau et al. 1999; Lhatoo et al. 2001; Ding et al. 2006).

A common cause of epilepsy-related deaths is SUDEP, defined as a sudden unexpected or witnessed or unwitnessed nontraumatic and nondrowning death in patients with epilepsy, with or without evidence of seizures and excluding documented status in which postmortem examination does not reveal a toxicological or anatomical cause of death. The reported incidence of SUDEP has ranged from 0.35 to 9.3 per 1000 person years depending on the different study populations and methodologies used (Hitiris et al. 2007; Jehi 2008). The incidence of death for young adults with intractable epilepsy is many times out of the general population with a peak between the ages of 20-40 years (Hitiris et al. 2007).

Status epilepticus - an emergency condition characterized by recurrent seizures and the patient not returning to their normal baseline-accounts for between $0.5 \%$ and $10 \%$ of all deaths in epilepsy with a standardized mortality ratio of 2.8 (Logroscino et al. 2002). People with epilepsy may sustain a fatal accident, either during a seizure or as a consequence of a seizure. Accidental deaths related to epilepsy are commonly caused by drowning, traffic accidents, trauma, falls, burns, or aspiration. Based on records from emergency departments, the risk of injury as a result of seizures was estimated to be 29.5 per 100,000 per year (Kirby and Sadler 1995). Accident-related deaths in people with epilepsy comprised $1.2 \%-6.5 \%$ of all deaths in community-based studies (Sillanpää et al. 1998; Lhatoo et al. 2001); 7.3\%-42\% in selected population studies with standard mortality ratios ranging from $2.4 \%$ to $5.6 \%$ (Institute of Medicine 2012). People with epilepsy have an increased risk of drowning with a standardized mortality ratio of 5.4 to 96.9 , which varies depending on the study population (Nilsson et al. 1997; Bell et al. 2008).

Common nonepileptic causes of death cited in mortality studies include neoplasms, stroke, hemorrhage, and pneumonia. Reports have noted decreased standardized mortality ratio for malignant neoplasia of the lungs, pancreas, hepatobiliary system, breasts, and lymphoid tissue (DeLorenzo et al. 1996). Cancer accounts for $16 \%-29 \%$ of deaths reported with standardized mortality ratios ranging from $3.4 \%$ to $5.4 \%$ (Lindsten et al. 2000; Shackleton et al. 2002; Bell et al. 2008).

\section{RISK FACTORS FOR EPILEPSY}

The risk factors for epilepsy in adults are somewhat established and are further discussed in the section, What Causes Epilepsy? There are several known risk factors for epilepsy in adults including head trauma, central nervous system (CNS) infections, such as neurocysticercosis, strokes, both embolic and hemorrhagic, CNS malignancies, particularly cortically based tumors, such as gliomas and metastatic lesions, Alzheimer's disease, and other neurodegenerative conditions. However, the relationship between epilepsy and other conditions, such as subcortical white matter diseases, demyelinating conditions, and certain psychiatric conditions (i.e., depression and schizophrenia), have not been sufficiently characterized.

\section{THE PROGNOSIS OF EPILEPSY}

Figure 1 (Jacoby and Baker 2008) outlines the possible courses that epilepsy may take in a given individual. 


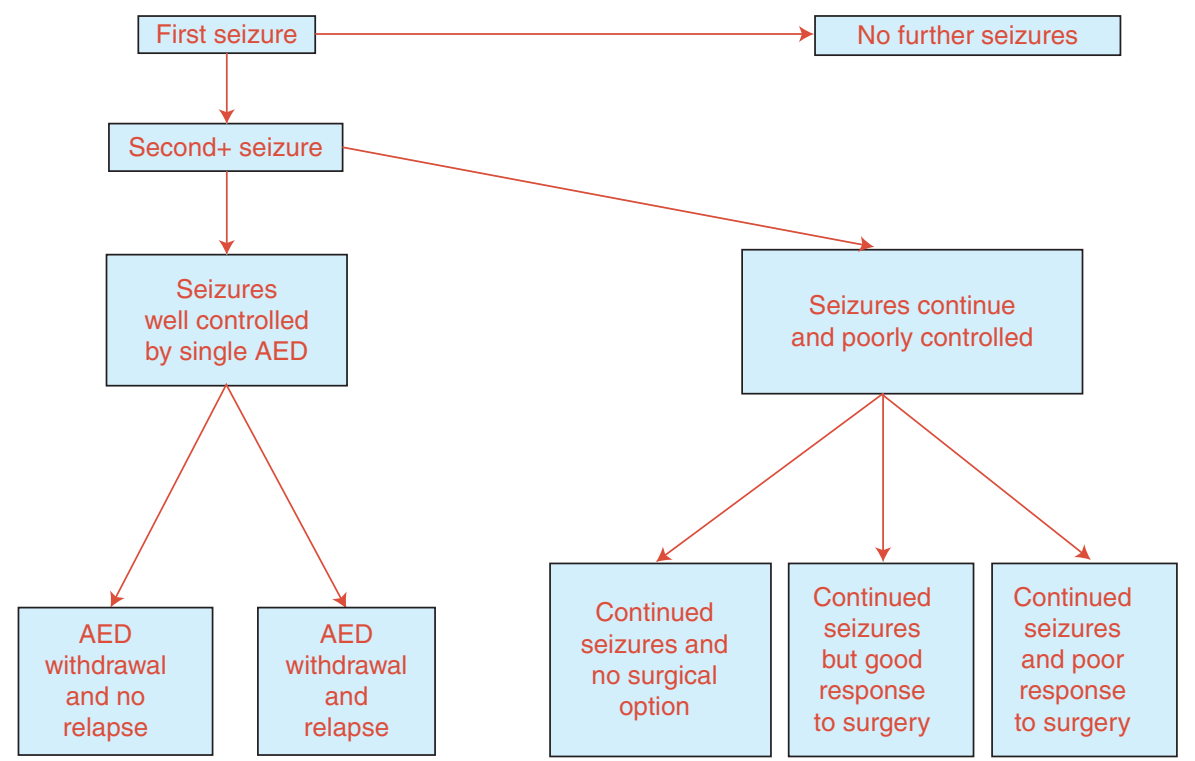

Figure 1. Possible courses of epilepsy. Outline of the potential courses that any given patient may take over the course of their lifetime with epilepsy, starting with the first seizure. AED, antiepileptic drug. (From Jacoby and Baker 2008; reprinted, with permission, from Elsevier (C) 2008.)

Generally speaking, individuals with epilepsy will fall into two large categories: (1) those that respond well to medication and their seizures are well controlled, or (2) those that fail to respond to medication are deemed drug resistant. It follows that identifying those individuals who will belong to the latter group is essential.

In 2009, the ILAE defined drug-resistant epilepsy as a failure of informative trials of two tolerated and appropriately chosen and used antiepileptic drug (AED) schedules, whether as monotherapies or in combination, to achieve sustained seizure freedom (Kwan et al. 2009). The manner in which the therapeutic intervention is deemed informative is related to a number of factors, including patient adherence to therapy, duration of exposure, inability to tolerate the medication, psychosocial concerns, administrative problems, access issues, and efforts to optimize dose. In one study (Berg and Kelly 2006), early-onset localization-related epilepsy might not become clearly intractable for many years. In the study, 333 patients were evaluated for resective surgery for localization-related epilepsy that was prospectively identified at the initial evaluation. Latency from epilepsy onset to failure of two AEDs was almost 9.1 years. Twenty-six percent of this group reported at least 1 year of remission and $8.5 \%$ of the group reported a 5 -year remission rate (Berg and Kelly 2006).

The percentage of individuals deemed intractable range from $9 \%$ to $26 \%$ depending on the studied population. Moreover, the natural history of untreated epilepsy is unknown. AEDs with some level of effectiveness have been available now for almost a century. There have been observational data from low-income countries where, in the majority of patients who receive no treatment, spontaneous remissions could approach up to $30 \%$. In contrast, formal epidemiologic studies have found that patients who have had at least two seizures have a very low chance of becoming seizure free. AEDs may delay seizure recurrence and reduce frequency, but do not appear to affect the long-term epilepsy development or prognosis.

Only $5 \%-10 \%$ of all incident cases of epilepsy ultimately result in truly intractable disease. These cases probably account for half of the cases of epilepsy (Berg and Kelly 2006; 
J.I. Sirven

Kwan et al. 2009). Approximately $60 \%$ of patients with intractable epilepsy can be expected to suffer from partial seizures (Kwan et al. 2009). Studies suggest that failure to control seizures with the first or second antiepileptic drug implies that the probability of subsequent seizure control with further AEDs is only $\sim 4 \%$. Recent studies reported that $\sim 5 \%$ of patients per year with intractable epilepsy were seizure free for 12 months following medication changes (Kwan and Brodie 2006). The finding highlights the fact that, irrespective of the number of previous AEDs, there is still a small possibility of inducing meaningful seizure remission in the population.

\section{WHAT CAUSES EPILEPSY?}

In 1989, the ILAE divided epilepsy etiology into idiopathic, cryptogenic, and symptomatic groups. Idiopathic epilepsies were presumed genetic, cryptogenic epilepsies were likely to have a cause, and symptomatic epilepsies had an identifiable cause (Commission 1989). It is important to understand that the disease of epilepsy is a final common pathway for numerous pathophysiological processes.

\section{IDIOPATHIC EPILEPSY}

Idiopathic epilepsies constitute $40 \%$ of the epilepsies worldwide (Shorvon et al. 2011). They are characterized by generalized or partial seizures in otherwise normal individuals and no prior history of seizures and a normal medical history. Response to AEDs is satisfactory, but it is unproven that treatment changes occur. Most cases of idiopathic epilepsy show a complex pattern of inheritance in which various genes act in different ways in each patient to produce the specific phenotype or syndrome. The various syndromes of idiopathic epilepsy differ in age of onset. Idiopathic epilepsies with a complex polygenic inheritance are divided into the idiopathic generalized epilepsies and the idiopathic partial epilepsies of childhood.

A few idiopathic epilepsy syndromes have a familial distribution with simple inheritance, usually autosomal dominant with reduced penetrance, and are usually caused by mutations in single genes that encode for iron channels or their accessory subunits. This has led to the concept that idiopathic epilepsies are channelopathies even though the link between molecular deficit and clinical phenotype is still insufficiently characterized, and non-ion channel genes are now emerging as causes of sporadic or familial early-onset, focal, and seemingly idiopathic seizure disorders. It is notable that mutations in the same gene can cause different epilepsy syndromes, and the same syndrome can be caused by mutations in different genes; therefore, there is considerable phenotypic and genotypic heterogeneity adding to the complexity.

\section{CRYPTOGENIC/SYMPTOMATIC EPILEPSIES}

Symptomatic epilepsies are often associated with structural brain abnormalities indicating an underlying disease or condition. This category includes developmental and congenital disorders associated with genetic or acquired cerebral pathological changes to acquired conditions. In symptomatic epilepsies, the underlying genetic conditions are responsible for either clear neuropathological abnormalities, such as in neurocutaneous diseases, or more subtle changes at the molecular level, such as Angelman syndrome, Rett syndrome, and others.

\section{Symptomatic Epilepsies Caused by Genetic or Congenital Disorders}

These include single gene disorders in that these disorders cause epilepsy and manifest primarily in childhood. Seizures are only one symptom of a much broader clinical picture characterized by learning disability and other features.

\section{Chromosomal Disorders}

Epilepsy is frequently observed in chromosomal disorders. These syndromes are associated with behavioral and intellectual disabilities and characteristic dysmorphic features. Examples of this include Angelman syndrome, ring chromosome 20 syndrome, and $4 \mathrm{P}$ syndrome. 


\section{Inherited Metabolic and Mitochondrial Disorders}

Seizures are often part of the clinical pictures of inherited metabolic and mitochondrial disorders particularly when these conditions begin early in life. Unfortunately, the clinical presentation in seizures is seldom distinctive enough to allow immediate diagnosis. Clinical phenotypes, epilepsy syndromes, and especially the characteristic times of presentation narrow diagnostic possibilities. The electroencephalogram (EEG) may also help facilitate recognition of the more common diagnoses. All infants and young children seen with unexplained epilepsy and neurological disability should be evaluated for inherited metabolic disorders. A positive family history may provide that important clue.

\section{Malformations of Cortical Development}

Malformations of cortical development include a wide range of disorders that commonly cause neurodevelopmental delay in epilepsy, especially in children. They account for at least 40\% of drug-resistant childhood epilepsies (Palmini 2011; Barkovich et al. 2012; Palmini et al. 2013). Abnormalities of neuronal and glial proliferation of programmed cell death, neuronal migration, synaptogenesis, and cortical organization result in distinct or partially overlapping entities. Some malformations are associated with mutations of specific genes but others result from environmental influences such intrauterine infection or profusion failure.

The most common malformation of cortical development is focal cortical dysplasia. This term designates a spectrum of abnormalities of the laminar structure of the cortex variably associated with cytopathological features including giant cell neurons, dysmorphic neurons, and balloon cells. The most frequent presentation of focal cortical dysplasia is a child or adolescent developing intractable partial epilepsy. However, infantile spasms may be the first manifestation. Focal cortical dysplasia is a frequent cause of focal status epilepticus and is the most common pathological substrate in epilepsy surgery series reaching up to $40 \%$ (Palmini 2011;
Barkovich et al. 2012; Palmini et al. 2013). Focal cortical dysplasia is rarely a restricted process as surface and intracranial EEG recordings reveal focal positive spike discharges or high-frequency discharges.

\section{Neurocutaneous Disorders}

The neurocutaneous disorders are a group of distinct conditions characterized by congenital dysplastic abnormalities involving the skin and nervous system. Tuberous sclerosis complex is a dominant disorder manifested primarily by abnormalities of the CNS, the skin, and the kidney. About $80 \%$ of tuberous sclerosis patients develop epilepsy with about two-thirds presenting with seizures before the age of 2 years, often with infantile spasms (Orlova and Crino 2010). Sturge-Weber syndrome is a nonfamilial neurocutaneous syndrome in which a venous angioma of the leptomeninges is accompanied by ipsilateral nevus flammeus of the skin supplied by the trigeminal nerve. Seventy percent of these patients develop seizures within the first year of life and almost all have epilepsy before 4 years of age (Comi 2011).

\section{Symptomatic Epilepsies Caused by Acquired Conditions}

Numerous symptomatic epilepsies are attributed to acquired conditions. These include primary cerebral pathological processes as well as epilepsies caused by external or environmental causes. Seizures can result from anything that injures the brain with the types of injury depending on age. Seizures in children often are caused by birth trauma, infections such as meningitis, and congenital abnormalities. Nonaccidental brain injury, often underrecognized, is a relatively rare cause of symptomatic epilepsy in children. In middle years, head injuries, infectious etiologies, alcohol, stimulant drugs, and medication side effects commonly cause seizures. In older adults, a higher proportion of seizures are cause by brain tumors and cerebrovascular disease. Here are some important acquired conditions that often cause epilepsy. 
J.I. Sirven

\section{Cerebral Palsy (CP)}

$\mathrm{CP}$ is associated with epilepsy in $\sim 50 \%$ of quadriplegic and hemiplegic forms and $\sim 26 \%$ of spastic diplegic and in dyskinetic forms (Guerrini 2006). Infantile spasms are observed in $>15 \%$ of patients (Guerrini 2006). Epilepsy often has an early onset and may be focal or generalized and with a variable course.

\section{Hippocampal Sclerosis}

The term, hippocampal sclerosis, designates subtle different entities featuring gliosis and neuronal loss in temporal structures. There are several pathologic substrates that are associated with additional pathological changes and in some cases outside the hippocampus. Typical magnetic resonance imaging (MRI) features of hippocampal sclerosis are reduced following increased signal intensity on T2 weighted imaging and abnormal morphology of hippocampus. There is no sex or side preference. Forty-eight to fifty-six percent of cases are bilateral in postmortem studies. In children with temporal lobe epilepsy, MRI detected hippocampal sclerosis in $21 \%$ of those with new-onset seizures and 57\% of those with refractory seizures. Thus, hippocampal sclerosis is the most common pathological finding in mesial temporal epilepsy, accounting for $\sim 70 \%$ of patients undergoing surgery for drug-resistant focal seizures. The clinical features include early initial injury, typically complicated febrile convulsions, but also CNS infections or head injury, followed by a variable latent interval of $\sim 7-10$ years between the onset of seizures in early childhood or adolescence.

\section{Cerebrovascular Disease}

Cerebrovascular disease often causes both acute symptomatic seizures and epilepsy. Seizures are both a common complication of ischemic stroke and can even be a presenting symptom. Early seizures occur within 7 days of stroke symptoms. Late seizures occur thereafter.

Intracranial hemorrhage, a form of cerebrovascular disease, is a frequent cause of epilepsy and often the result of a penetrating head injury. Nontraumatic intracranial hemorrhages are more prominent in children and young adults and more common among men than women. Causes for nontraumatic intracerebral hemorrhage are aneurysms, vascular malformations, venous angiomas, cavernous angiomas, telangiectasias, acute or chronic hypertension, amyloid angiopathy, venous thrombosis, primary and metastatic brain tumor, vasculitis, and bleeding disorders. Vascular malformations may cause epilepsy even in the absence of overt bleeding. Cavernous angiomas and arteriovenous malformations are the most epileptogenic lesions. About $50 \%-70 \%$ of seizures occur within the first 24 hours of intracranial hemorrhage and $90 \%$ in the first 3 days with an overall 30-day risk of seizures being $8 \%$ (Menon and Shorvon 2009).

\section{Neoplasms}

Brain tumors account for $3.6 \%$ of all epilepsy and $12 \%$ of all acquired epilepsy (Lynam et al. 2007; Van Breemen et al. 2007). Epilepsy from brain tumors occurs at all ages, but a higher proportion in the 25- to 64-year-old age group. Seizures are the most common presenting symptoms of primary brain tumors. Some patients have one seizure leading to the tumor diagnosis and will never have another seizure after the tumor is resected. In other types of brain tumors, such as dysembrioplastic neuroepithelial tumors, the natural course of the tumor is very benign, but the severity of epilepsy dictates the necessity for surgical intervention. Seizure incidence is low in some of the most malignant brain tumors, such as glioblastoma and primary CNS lymphoma, and higher in some less-malignant infiltrative lesions, such as WHO grade 2 diffuse gliomas. Location in the temporal lobe and cortical rather than white matter involvement are usually associated with a higher risk of seizures.

\section{Head Trauma}

Brain injury accounts for $6 \%$ of all epilepsy and occurs in all ages (Temkin et al. 1990; Christen- 
Epilepsy: A Spectrum Disorder

sen et al. 2009). Posttraumatic seizures are traditionally subdivided into immediate, early, and late seizures. Early seizures increase the risk of posttraumatic epilepsy. Several processes contribute to the development of early seizures including hypoxia, increased intracranial pressure, hypotension, edema, ischemia, electrolyte imbalance, and infection. Late seizures reflect permanent neuropathological changes in the brain and tend to recur, therefore, signaling the onset of posttraumatic epilepsy.

\section{Cerebral Infections}

Acute symptomatic seizures defined by the ILAE as occurring within 7 days of an acute CNS infection are seen in $2 \%-67 \%$ of patients with encephalitis (Singhi 2011). The incidence of acute symptomatic seizures and subsequent epilepsy varies with the encephalitis. The risk of developing chronic epilepsy is highest within the first 5 years following encephalitis, but it can occur up to 20 years later (Singhi 2011). The most common serious viral encephalitis is herpes simplex virus type 1 , and this infection often results in severe and intractable epilepsy. It is important to make an early diagnosis. Other viruses that need to be considered include varicella zoster virus and enterovirus. Pyogenic brain abscess is another uncommon, but serious infection that causes epilepsy. Streptococci organisms are often isolated, but many others are also noted. It is of note that neurocysticercosis is the most common parasitic disease of the CNS and a major cause of epilepsy in endemic areas, such as Mexico, India, and China (Singhi 2011). In Africa, malaria is a particularly common cause of seizures and, typically, status epilepticus is the acute phase of cerebral malaria. There is a 9- to 11-fold increase in risk of chronic epilepsy in children with a history of malaria. Epilepsy is also frequently a presenting symptom of cerebral tuberculoma (Singhi 2011).

\section{Immunologic and Inflammatory Diseases}

Epilepsy may occur in a number of autoimmune or inflammatory disorders. Clinical pre- sentation varies among patients and includes seizures, psychiatric features, movement disorders, amnesia, confusion, or loss of consciousness. These diseases are often associated with tumors and they are more often nonparaneoplastic and immunotherapy is often effective. Some conditions, such as limbic encephalitis, an anti-NMDA receptor encephalitis, can be a very epileptogenic condition, and immunotherapy is the approach that one takes in management of these conditions. Epilepsy can occur in other forms of vasculitides, sometimes caused by cerebral infarctions.

\section{Neurodegenerative Diseases}

Degenerative dementias, such as Alzheimer's disease, are associated with an increased incidence of seizures. The prevalence of seizures in patients with dementia ranges from 10\% to $20 \%$ (Institute of Medicine 2012). Patients with seizures are younger and significantly more cognitively impaired than those with dementia only. Seizures also occur in prion disorders, frontal temporal lobar degeneration syndromes, whereas Parkinson's disease and other Parkinsonian conditions seem relatively seizure free.

\section{Reflex or Provoked Epilepsy}

Reflex or provoked epilepsy is a condition in which seizures can be provoked habitually by an external stimulus or, less commonly, by an internal mental process. Some individuals with reflex epilepsy may have seizures only with specific stimuli, or others may have both reflex and spontaneously occurring seizures. Simple reflex seizures are precipitated by elementary sensory stimuli, such as flashes of light or being startled, whereas complex reflex seizures are precipitated by patient-specific, more elaborate stimuli. Photosensitive epilepsy is the most common type of reflex epilepsy. Clinical photoconvulsive seizures with subclinical photoparoxysmal responses occur when an individual is exposed to visual stimuli, usually flashes of light of a particular frequency. It is believed that this provoked epilepsy is an inherited trait. 
J.I. Sirven

\section{QUALITY OF LIFE IN EPILEPSY}

The quality of life of people with epilepsy is directly related to a number of factors, including seizure frequency and severity, injuries, AED toxicity, and mood. The typical trajectory for quality of life in epilepsy is shown in Figure 2 (Jacoby and Baker 2008).

Seizure frequency is a significant inverse predictor of all facets of quality of life, in which increased seizure frequency leads to a poor quality of life. Perception of stigma by both adults and children with epilepsy is related to seizure frequency and severity. Depression, anxiety, and other psychiatric disorders are much more common in people with epilepsy than in the general population and may be more common than in other chronic disorders, such as asthma or diabetes. Patients with temporal lobe epilepsy appear to be more likely to suffer from depression than those with other seizure types. Patients with depression in addition to epilepsy have a reduced quality of life, lower income, and underemployment. Even when epilepsy is in remission, social difficulties seem to persist. People with epilepsy have lower educa- tional levels, lower marriage rates, and higher unemployment rates. They also seem to have altered reproductive function.

\section{PSYCHIATRIC AND COGNITIVE COMORBIDITIES}

\section{Psychiatric}

Psychiatric comorbidities are relatively frequent in people with epilepsy, having been identified in one out of every three patients in the course of their lives. The relationship between some of the psychiatric disorders in epilepsy is complex. For example, depressive disorders in epilepsy have been found to have a bidirectional relation. It is not only that those with epilepsy are at a greater risk of developing mood disorders, but patients with mood disorders are at a greater risk of developing epilepsy. Depressive and anxiety disorders are the most frequent psychiatric comorbidities in people with epilepsy and, more often than not, they tend to occur together. The observations have significant implications with respect to the evaluation and management of these comorbidities. Despite

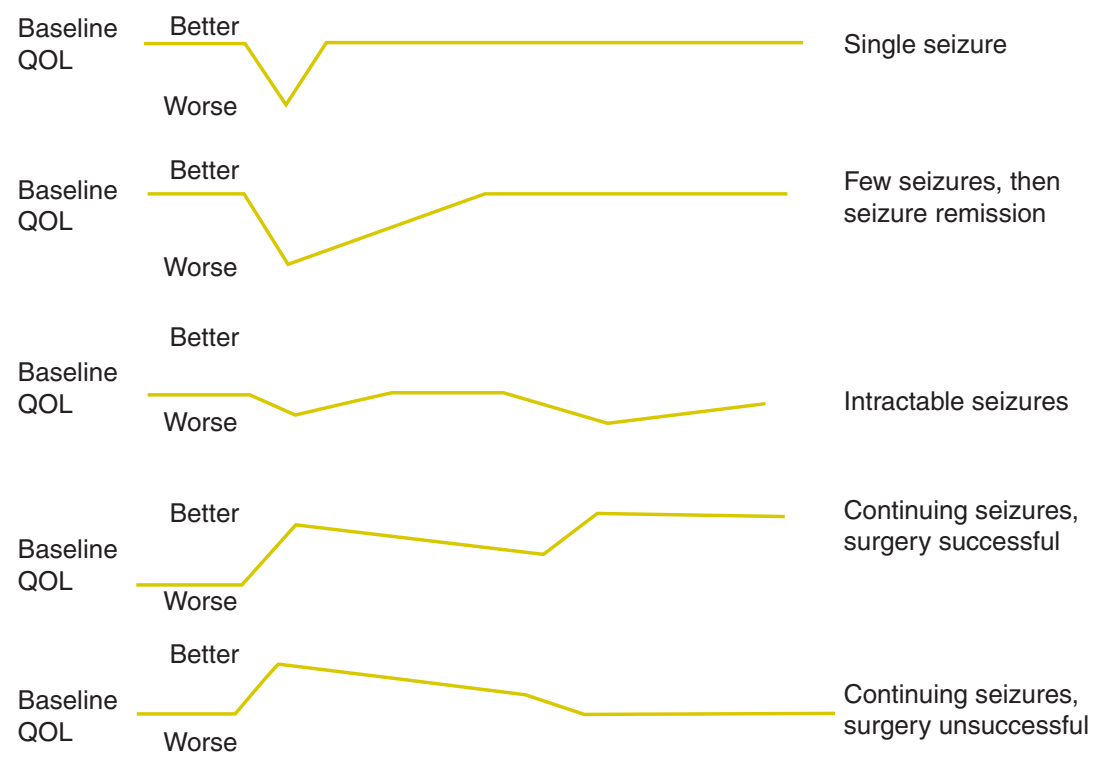

Figure 2. Quality of life (QOL) in epilepsy. The spectrum of possibilities for any given patient with seizures and the effect that the totality of the condition and its treatment has on the quality of life for patients with epilepsy. (From Jacoby and Baker 2008; reprinted, with permission, from Elsevier (C) 2008.) 
Epilepsy: A Spectrum Disorder

the relatively high frequency of psychiatric comorbidities, the available data on the clinical characteristics and treatment are limited based on the lack of high-quality evidence.

The prevalence rates of mood and anxiety disorders have ranged from $10 \%$ to $60 \%$ in the literature depending on the methodology (Tellez-Zenteno et al. 2007). Population studies indicate that one in three to four people with epilepsy have experienced a mood disorder in the course of their lives. The lifetime prevalence of mood disorders based on a Canadian study was $24 \%$ in people with epilepsy compared with $13 \%$ in controls, whereas $17 \%$ had a lifetime history of a major depressive disorder compared with $10 \%$ of controls, including dysthymia (Tellez-Zenteno et al. 2007). The lifetime prevalence rate of depressive disorders increased to $34.2 \%$ (Tellez-Zenteno et al. 2007). Population-based studies have identified a lifetime prevalence of anxiety disorders ranging from $11 \%$ to $30 \%$ (Tellez-Zenteno et al. 2007). In the same Canadian study, the lifetime prevalence rate was $22.8 \%$ in people with epilepsy versus $11.2 \%$ in nonepilepsy patients (TellezZenteno et al. 2007), whereas that of panic disorder or agoraphobia was $6.6 \%$ versus $3.6 \%$, respectively (Tellez-Zenteno et al. 2007).

Suicidal ideation, attempts, and completed suicide are a serious complication of mood and anxiety disorders. People with epilepsy had a twofold higher risk of completed suicide compared with nonepilepsy controls (Hesdorffer et al. 2006). In the absence of any psychosocial problem, the risk increased by 32 -fold in the presence of a mood disorder and by 12 -fold in the presence of the anxiety disorder (Hesdorffer et al. 2006).

\section{Cognitive}

Twenty to fifty percent of all patients with epilepsy report cognitive problems that can significantly impair daily activities and quality of life (Halgren et al. 1991; Vermeulen et al. 1993; Perrine and Kiolbasa 1999; Helmstaedter et al. 2004; Pugh et al. 2011; Kleen et al. 2012, 2013). The neurophysiology of seizures and interictal abnormalities, presence of underlying structural abnormal- ities, such as hippocampal sclerosis, and side effects of antiepileptic medications, can all contribute to cognitive impairment. In addition, mood is significantly associated with subjective cognitive complaints. Individuals with epilepsy perceive their memory to be more severe than the observed deficits when measured objectively.

Further complicating this situation is that treatment options are limited. Thus, managing this aspect of the epilepsy spectrum is vexing even to the most skillful clinician.

\section{LOOKING BEYOND EPIDEMIOLOGY: THE STATE OF EPILEPSY CARE IN THE UNITED STATES}

In 2005, the U.S. Centers for Disease Control and Prevention (CDC) conducted surveillance work to assess the state of epilepsy and seizure care in the United States based on 19 reporting states (Kobau et al. 2005). The CDC worked with the Behavioral Risk Factor Surveillance System and assessed a number of epilepsy and seizurerelated variables to better characterize how well the U.S. healthcare structure was handling epilepsy care. The Behavioral Risk Factor Surveillance System is an ongoing, state-based, random digit dial telephone survey of noninstitutionalized U.S. adults over the age of 18 . This system collects information on health risk, behaviors, and preventative health services that relate to the leading causes of death and morbidity.

The surveillance system included a total of 2207 adults from 19 states, and 1.65\% reported a history of epilepsy (Kobau et al. 2005). Moreover, $0.84 \%$ had active epilepsy defined as either a history of epilepsy and currently taking medications or reporting one or more seizures during the past 3 months; $0.75 \%$ were classified as having inactive epilepsy or a history of epilepsy or seizure disorder but not currently taking medicine to control epilepsy and no seizures in the 3 months preceding the survey (Kobau et al. 2005). There were no differences among the states with regards to prevalence of lifetime epilepsy, active epilepsy, or inactive epilepsy. Prevalence estimates for active and inactive epilepsy revealed no significant difference by sex, race, or ethnicity. 
J.I. Sirven

However, American adults with a history of epilepsy and active epilepsy were much more likely to report fair or poor health by being unemployed or unable to work (Kobau et al. 2005). These individuals also lived in households with the lowest annual incomes and had a history of concomitant disorders, such as stroke or arthritis. Adults with a history of epilepsy and active epilepsyalso reported significantly worse healthrelated quality of life. Individuals with epilepsy were more likely to be obese, physically inactive, and smokers. In adults with active epilepsy with recent seizures, $16.1 \%$ reported not taking their epilepsy medications and $65.1 \%$ reported having had more than one seizure in the past month (Kobau et al. 2005). Among adults with a history of epilepsy, almost $24 \%$ reported cost as a barrier to seeking care from a physician over the previous year (Kobau et al. 2005). A total of 35\% of adults with active epilepsy with seizures reported not having seen a neurologist or an epilepsy specialist in the previous year (Kobau et al. 2005).

This study showed that the incidence and prevalence rates that are reported by population-based studies are relatively accurate. Seizures and epilepsy are a frequent occurrence among the American population. Yet, the CDC analysis also showed the significant burden of the disease, which cannot be assessed from epidemiological studies. A number of public health interventions are necessary to better educate the public, understand the barriers to care, financing of the care, and how to improve quality of life for patients with epilepsy.

\section{TREATMENT AND RESPONSE}

There are now 24 AEDs approved for use in epilepsy in the United States by the Food and Drug Administration (FDA) along with two neuromodulation devices, several surgical procedures, and two diets. Diagnosis and therapy are discussed in the literature. The following is a general overview of overall treatment response.

\section{Medication Response}

About $70 \%$ of patients with any type of epilepsy will respond to any medication, whereas 30\% do not. About $10 \%$ of patients with an initial positive antiepileptic drug response cease to respond for a variety of physiological reasons. In one large randomized controlled study, Mattson and colleagues found that only a small number of patients who had not responded to monotherapy with two appropriate AEDs or a combination of two drugs would respond to a third (Mattson et al. 1992). Recent data using new AEDs also seem to confirm these findings (Kwan et al. 2011). Once a patient has failed to respond to two AEDs, the chance of achieving control in the third appears to be $<5 \%$. Thus, available data suggests that trying more than three AEDs, at most, is unlikely to help.

Even when using newer AEDs, only 60\% of all patients remained seizure free on AED. At least initial prognosis in $90 \%$ of patients could be determined within 1 to 2 years. Even though patients with an initial response might relapse, those who do not become seizure free on the first AEDs were very unlikely to do so with subsequent drug trials. The prognosis for AED control of partial seizure disorders may be particularly poor. In the Veterans Administration Cooperative Study, only $20 \%$ to $30 \%$ of patients with complex partial seizures achieve seizure freedom compared with $60 \%-70 \%$ of generalized tonic-clonic seizures (Mattson et al. 1992).

Data on the treatment of Lennox-Gastaut syndrome, infantile spasms, and other catastrophic epilepsies, however, are limited. These epilepsies are intractable almost by definition, as most currently available drugs have limited efficacy. Infantile spasms, in particular, are refractory in almost $50 \%$ of cases.

Dietary therapy is limited by the difficulty in patient compliance. Most studies assessing this treatment reveal a response of $30 \%$ to $33 \%$ seizure control, 33\% seizure reduction, and 33\% have no response (Sirven et al. 1999; Kossoff et al. 2008) for all types of epilepsy.

\section{Surgery Response}

Surgery offers the best hope for altering the course of epilepsy. There has only been one controlled study comparing medical and surgical prognosis. Patients older than 16 years with 
temporal-lobe onset of seizures who had at least one seizure per month on two effective AEDs were randomized to either temporal lobectomy or optimization of medical therapy. At 1 year, $58 \%$ of surgical and $8 \%$ of medical patients were free of seizures impairing consciousness (Wiebe et al. 2001). Two patients who had undergone surgery had a decline in verbal memory that affected their work, one medical patient died from SUDEP. Other uncontrolled studies have shown similar outcomes.

Several studies have found evidence for progressive cognitive impairment in epilepsy, particularly in memory that may be associated with baseline imaging abnormalities, lower baseline function, and longer epilepsy duration. Patients with epilepsy may continue to have social and psychological problems, even when seizures are controlled. Cognitive dysfunction may persist as well, and it may be unclear how much is caused by subclinical seizures, AED therapy, or underlying brain dysfunction. Successful surgery may arrest or even reverse cognitive decline, although patients who continue to have frequent seizures may experience increased neuropsychological deficits.

Successful surgery tends to relieve depression and anxiety. In a multicenter study preoperatively, $22 \%$ of patients had moderate to severe depression and $25 \%$ anxiety. At 2 years of follow-up, prevalence was $17.6 \%$ and $14.7 \%$ of patients who continued to have postoperative seizures compared with $8.2 \%$ of those who were not seizure free (Engel et al. 2012). Two years after surgery, children report a significant improved social competence and self-worth; adolescents reported improved athletic competence and romance.

Neuromodulatory treatment with stimulation of the vagus nerve, trigeminal nerve, closed loop devices of cortical stimulation, and deep brain stimulation of the anterior thalamus are, at best, palliative therapies promising seizure reduction in $40 \%$ of patients with long-term therapy (Vagus Nerve Stimulation Study Group 1995; DeGiorgio et al. 2000, 2013; Fisher et al. 2010; Morrell 2011). Seizure freedom is rare and elusive with these devices. Nevertheless, they may provide some improvements in quality of life by avoiding common cognitive and psychiatric adverse effects associated with antiepileptic medications.

\section{CONCLUSION}

Epilepsy is a common complex spectrum disorder that can impact individuals of all ages, with a particular predilection for the young and the old. The disease has a multitude of phenotypic presentations and its causes are multifactorial ranging from monogenic etiologies to numerous cortical acquired pathologies. The severity of the condition can vary widely among patients with significant morbidity and mortality. Despite the numerous medical, surgical, immunological, neuromodulatory, and dietary therapies available to help control seizures, public health surveys continue to show that a considerable number of those afflicted have poor quality of life because of the impact that recurrent seizures have on activities of daily life.

\section{ACKNOWLEDGMENTS}

The author has no conflict of interest or financial involvement with this manuscript and acknowledges the Mayo Foundation for Medical Education and Research.

\section{REFERENCES}

Annegers JF, Hauser W, Shirts S. 1984. Heart disease mortality and morbidity in patients with epilepsy. Epilepsia 25: 699-704.

Aziz H, Ali S, Frances P, Khan MI, Hasan KZ. 1994. Epilepsy in Pakistan: A population-based epidemiologic study. Epilepsia 35: 950-958.

Barkovich A, Guerrini R, Kuzniecky RI, Jackson GD, Dobyns WB. 2012. A developmental and genetic classification for malformation of cortical development. Brain 135: $1348-1369$.

Begley C, Famulari M, Annegers J, Lairson D, Reynolds T, Coan S, Dubinsky S, Newmark ME, Leibson C, So EL, et al. 2000. The cost of epilepsy in the United States: An estimate from population based clinical and survey data. Epilepsia 42: 1553-1562.

Bell GS, Sander JW. 2001. The epidemiology of epilepsy: The size of the problem. Seizure 10: 306-314.

Bell GS, Gaitatzis A, Bell CL, Johnson A Sander JW. 2008. Drowning in people with epilepsy; How great is the risk? Neurology 71: 578-582. 
J.I. Sirven

Benn EK, Hauser WA, Shih T, Leary L, Bagiella E, Dayan P, Green R, Andrews H, Thurman DJ, Hesdorffer DC. 2008. Estimating the incidence of first unprovoked seizure and newly diagnosed epilepsy in the low-income urban community of Northern Manhattan. Epilepsia 49: 14311439.

Berg AT, Kelly MM. 2006. Defining intractability: Comparisons among published definitions. Epilepsia 47: 431436.

Berg A, Berkovic S, Brodie MJ, Buchalter J, Cross JH, van Emde Boas W, Engel J, French J, Glauser TA, Mathern GW, et al. 2010. Revised terminology and concepts for organization of seizures and epilepsies: Report of the ILAE Commission on Classification and Terminology, 2005-2009. Epilepsia 51: 676-685.

Brewis M. 1966. Neurological disease in an English city. Munksgaard, Copenhagen.

Camfield CS, Camfield PR, Veugelers PJ. 2002. Death in children with epilepsy: A population based study. Lancet 359: 1891-1895

Christensen J, Pedersen MG, Pedersen CB, Sidenius P, Olsen J, Vestergaard M. 2009. Long term risk of epilepsy after traumatic brain injury in children and young adults: A population based cohort study. Lancet 373: 1105-1110.

Cockerell OC, Johnson AL, Sander JW, Hart YM, Goodridge DM, Shorvon SD. 1994. Mortality from epilepsy: Results from a prospective population-based study. Lancet 344: 918-921.

Comi A. 2011. Presentation, diagnosis, pathophysiology and treatment of the neurological features of Sturge-Weber syndrome. Neurologist 17: 179-184

Commission on Classification and Terminology of the ILAE. 1989. Proposal for revised classification of epilepsies and epileptic syndromes. Epilepsia 30: 389-399.

DeGiorgio CM, Schachter SC, Handforth A, Salinsky M Thompson J, Uthman B, Reed R, Collins S, Tecoma E, Morris GL, et al. 2000. Prospective long-term study of vagus nerve stimulation for the treatment of refractory seizures. Epilepsia 41: 1195.

DeGiorgio CM, Soss J, Cook IA, Markovic D, Gornbein J, Murray D, Oviedo S, Gordon S, Corralle-Leyva G, Kealey CP, et al. 2013. Randomized controlled trial of trigeminal nerve stimulation for drug-resistant epilepsy. Neurology 80: 786-791.

De Graaf A. 1974. Epidemiological aspects of epilepsy in Northern Norway. Epilepsia 15: 291-299.

DeLorenzo RJ, Hauser WA, Towne AR, Boggs JG, Pellock JM, Penberthy L, Garnett L, Fortner CA, Ko D. 1996. A prospective population based epidemiologic study of status epilepticus in Richmond, Virginia. Neurology 46: 1029-1035.

Ding D, Wang W, Wu J, Ma G, Dai X, Yang B. 2006. Premature mortality in people with epilepsy in rural China: A prospective study. Lancet Neurol 5: 823-827.

Engel J, McDermott MP, Wiebe S, Langfitt JT, Stern JM, Dewar S, Sperling MR, Gardiner I, Erba G, Fried I, et al. 2012. Early surgical therapy for drug-resistant temporal lobe epilepsy: A randomized trial. JAMA 307: $922-$ 930.

Fisher R, van Emde Boas W, Blume W, Elger C, Genton P, Lee P, Engel J Jr. 2005. Epileptic seizures and epilepsy: Defi- nitions proposed by the International League Against Epilepsy (ILAE) and the International Bureau for Epilepsy (IBE). Epilepsia 46: 470-472.

Fisher R, Salanova V, Witt T, Worth R, Henry T, Gross R, Oommen K, Osorio I, Nazzaro J, Labar D, et al. 2010. Electrical stimulation of the anterior nucleus of thalamus for treatment of refractory epilepsy. Epilepsia 51: 899_ 908.

Fisher RS, Acevedo C, Arzimanoglou A, Bogacz A, Cross JH, Elger CE, Engel J Jr, Forsgren L, French JA, Glynn M, et al. 2014. ILAE official report: A practical clinical definition of epilepsy. Epilepsia 55: 475-482.

Guerrini R. 2006. Epilepsy in children. Lancet 367: 499-524.

Halgren E, Stapleton J, Domalski P, Swartz BE, DelgadoEscueta AV, Walsh GO, Mandelkern M, Blahd W, Ropchan J. 1991. Memory dysfunction in epilepsy patients as a derangement of normal physiology. Adv Neurol 55: 385-410.

Harvey AS, Nolan T, Carlin JB. 1993. Community-based study of mortality in children with epilepsy. Epilepsia 34: 597-603.

Hauser WA, Annegers J, Elveback LR. 1980. Mortality in patients with epilepsy. Epilepsia 21: 399-412.

Helmstaedter C, Sonntag-Dillender M, Hoppe C, Elger CE. 2004. Depressed mood and memory impairment in temporal lobe epilepsy as a function of focus lateralization and localization. Epilepsy Behav 5: 696-701.

Hesdorffer DC, Hauser WA, Annegers JF, Cascino G. 2006. Depression and attempted suicide as risk factors for incident unprovoked seizures and epilepsy. Ann Neurol 59: $35-41$.

Hirtz D, Thurman DJ, Gwinn-Hardy K, Mohamed M, Chaudhuri AR, Zalutsky R. 2007. How common are the common neurologic disorders? Neurology 68: 326337.

Hitiris N, Suratman S, Kelly K, Stephen LJ, Sills GJ, Brodie MJ. 2007. Sudden unexpected death in epilepsy: A search for risk factors. Epilepsy Behav 10: 138-141.

Institute of Medicine Committee on the Public Health Dimensions of the Epilepsies. 2012. Epilepsy across the spectrum, pp. 19-47. National Academies, Washington, DC.

Jacoby A, Baker G. 2008. Quality of life trajectories in epilepsy: A review of the literature. Epilepsy Behav 12: 148 161.

Jehi L, Najm I. 2008. Sudden unexpected death in epilepsy: Impact, mechanisms, and prevention. Clev Clin J Med 75 (Suppl): S66-S70.

Kirby S, Sadler R. 1995. Injury and death as a result of seizures. Epilepsia 36: 25-28.

Kleen JK, Scott RC, Lenck-Santini P, Holmes G. 2012. Cognitive and behavioral co-morbidities of epilepsy. In Jasper's basic mechanisms of the epilepsies, 4th ed. (ed. Noebels JL, et al.). National Center for Biotechnology Information, Bethesda, MD.

Kleen JK, Scott RC, Holmes GL, Roberts DW, Rundle MM, Testorf M, Lenck-Santini PP, Jobst BC. 2013. Hippocampal interictal epileptiform activity disrupts cognition in humans. Neurology 17: 17.

Kobau R, Zahran H, Thurman D, Zack M, Henry T, Schachter S, Price P. 2005. Epilepsy surveillance among adults-19 
states. Behavioral Risk Factors Surveillance Risk System, www.cdc.gov/mmwr/preview/mmwrhtml/ss5706al.htm.

Kossoff EH, Rowley H, Sinha SR, Vining EP. 2008. A prospective study of the modified Atkins diet for intractable epilepsy in adults. Epilepsia 49: 316.

Kurtz Z, Tookey P, Ross E. 1998. Epilepsy in young people: 23 year follow-up of the British national child development study. BMJ 316: 339-342.

Kwan P, Brodie M. 2006. Issues of medical intractability for surgical candidacy. In The treatment of epilepsy practice (ed. Wyllie E, Gupta A, Lachhwani D), pp. 983. Lippincott, Williams \& Wilkins, Philadelphia.

Kwan P, Arzimanoglou A, Berg AT, Brodie MJ, Hauser WA, Mathern G, Moshé SL, Perucca E, Wiebe S, French J. 2009. Definition of drug resistant epilepsy: Consensus proposal by the ad hoc Task Force of the ILAE Commission on Therapeutic Strategies. Epilepsia 51: 1069-1077.

Kwan P, Schachter SC, Brodie MJ. 2011. Drug-resistant epilepsy. N Engl J Med 365: 919.

Lavados J, Germain L, Morales A, Campero M, Lavados P. 1992. A descriptive study of epilepsy in the district of El Salvador, Chile 1984-1988. Acta Neurol Scand 85: 249 256.

Lhatoo SD, Johnson AL, Goodridge DM, MacDonald BK, Sander JW, Shorvon SD. 2001. Mortality in epilepsy in the first 11 to 14 years after diagnosis: Multivariate analysis of a long-term, prospective, population-based cohort. Ann Neurol 49: 335-344.

Lindsten H, Nyström L, Forsgren L. 2000. Mortality risk in an adult cohort with a newly diagnosed unprovoked epileptic seizure. Epilepsia 41: 1469-1473.

Logroscino G, Hesdorffer DC, Cascino GD, Annegers JF, Bagiella E, Hauser WA. 2002. Long-term mortality after a first episode of status epilepticus. Neurology 58: 537541.

Loiseau J, Picot MC, Loiseau P. 1999. Short term mortality after a first epilepstic seizure; a population based study. Epilepsia 40: 1388-1392.

Lynam LM, Lyons MK, Drazkowski JF, Sirven JI, Noe KH, Zimmerman RS, Wilkens JA. 2007. Frequency of seizures in patients with newly diagnosed brain tumors: A retrospective review. Clin Neurol Neurosurg 109: 634-638.

Mattson RH1, Cramer JA, Collins JF. 1992. A comparison of valproate with carbamazepine for the treatment of complex partial seizures and secondarily generalized tonicclonic seizures in adults. The Department of Veterans Affairs Epilepsy Cooperative Study No. 264 Group. N Engl J Med 327: 765-771.

Menon B, Shorvon S. 2009. Ischaemic stroke in adults and epilepsy. Epilepsy Res 87: 1-11.

Morrell MJ; RNS System in Epilepsy Study Group. 2011 Responsive cortical stimulation for the treatment of medically intractable partial epilepsy. Neurology 13: 12951304.

Nashef L, Fish DR, Sander JW, Shorvon SD. 1995. Incidence of sudden unexpected death in an adult outpatient cohort with epilepsy at a tertiary epilepsy center. J Neurol Neurosurg Psychiatry 58: 462-464.

Nilsson L, Tomson T, Farahmand BY, Diwan V, Persson PG. 1997. Cause specific mortality in epilepsy: A cohort study of more than 9,000 patients once hospitalized for epilepsy. Epilepsia 38: 1062-1068.

Orlova J, Crino P. 2010. The tuberous sclerosis complex. Ann NY Acad Sci 1184: 87-105.

Palmini A. 2011. Revising the classification of focal cortical dysplasias. Epilepsia 52: 188-190.

Palmini A, Najm I, Avanzini G, Babb T, Guerrini R, Foldvary-Schaefer N, Jackson G, Lüders HO, Prayson R, Spreafico R, et al. 2004. Terminology and classification of the cortical dysplasias. Neurology 62: S2-S8.

Palmini A, Paglioli E, Silva VD. 2013. Developmental tumors and adjacent cortical dysplasia: Single or dual pathology? Epilepsia 54: 18-24.

Perrine K, Kiolbasa T. 1999. Cognitive deficits in epilepsy and contribution to psychopathology. Neurology 53: S39-S48.

Placencia M, Shorvon S, Paredes V, Bimos C, Sander JW, Suarez J, Cascante SM. 1992. Epileptic seizures in an Andean region of Ecuador. Incidence and prevalence and regional variation. Brain 115: 771-782.

Pugh MJ, Berlowitz DR, Rao JK, Shapiro G, Avetisyan R, Hanchate A, Jarrett K, Tabares J, Kazis LE. 2011. The quality of care for adults with epilepsy: An initial glimpse using the QUIET measure. BMC Health Serv Res 11: 1.

Sander JW. 2003. The epidemiology of epilepsy revisited. Curr Opin Neurol 16: 165-170.

Sander JW, Shorvon SD. 1996. Epidemiology of epilepsy: The size of the problem. J Neurol Neurosurg Psychiatry 61: 433-443.

Shackleton DP, Westendorp RG, Trenité DG, Vandenbroucke JP. 1999. Mortality in patients with epilepsy: 40 years of follow up in a Dutch cohort study. J Neurol Neurosurg Psychiatry 66: 636-640.

Shackleton DP, Westendorp RG, Kasteleijn-Nolst Trenité DG, de Craen AJ, Vandenbroucke JP. 2002. Survival of patients with severe epilepsy: An estimate of mortality risk. Epilepsia 43: 445-450.

Shorvon S, Andermann F, Guerrini R. 2011. The causes of epilepsy: Common and uncommon causes in adults and children. Cambridge University Press, Cambridge.

Sillanpää M, Jalava M, Kaleva O, Shinnar S. 1998. Longterm prognosis of seizures with onset in childhood. $N$ Engl J Med 338: 1715-1722.

Singhi P. 2011. Infectious causes of seizures and epilepsy in the developing world. Dev Med Child Neurol 53: 600609.

Sirven J, Whedon B, Caplan D, Liporace J, Glosser D, O’Dwyer J, Sperling MR. 1999. The ketogenic diet for intractable epilepsy in adults: Preliminary results. Epilepsia 40: 1721.

Tellez-Zenteno J, Patten S, Jette N, Williams J, Wiebe S. 2007. Psychiatric comorbidity in epilepsy a population based analysis. Epilepsia 48: 2336-2344.

Temkin NR, Dikmen SS, Wilensky AJ, Keihm J, Chabal S, Winn HR. 1990. A randomized, double-blind study of phenytoin for the prevention of post-traumatic seizures. N Engl J Med 323: 497-502.

Vagus Nerve Stimulation Study Group. 1995. A randomized controlled trial of chronic vagus nerve stimulation for treatment of medically intractable seizures. Neurology 45: 224 . 


\section{J.I. Sirven}

Van Breemen MS1, Wilms EB, Vecht CJ. 2007. Epilepsy in patients with brain tumours: Epidemiology, mechanisms, and management. Lancet Neurol 6: 421-430.

Vermeulen J, Aldenkamp AP, Alpherts WC. 1993. Memory complaints in epilepsy: Correlations with cognitive performance and neuroticism. Epilepsy Res 15: 157-170.
Wiebe S, Blume WT, Girvin JP, Eliasziw M; Effectiveness and Efficiency of Surgery for Temporal Lobe Epilepsy Study Group. 2001. A randomized, controlled trial of surgery for temporal-lobe epilepsy. N Engl J Med 345: 311.

Zielinski J. 1974. Epilepsy and mortality rate and cause of death. Epilepsia 15: 191-201. 


\section{$\&_{\mathrm{CSH}}^{\infty} \&$ Cold Spring Harbor

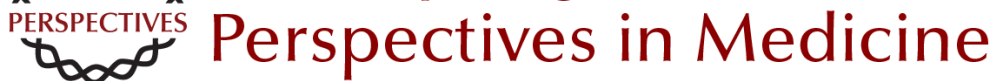

\section{Epilepsy: A Spectrum Disorder}

Joseph I. Sirven

Cold Spring Harb Perspect Med 2015; doi: 10.1101/cshperspect.a022848

Subject Collection Epilepsy: The Biology of a Spectrum Disorder

The Epilepsy Spectrum: Targeting Future Research Challenges

Gregory L. Holmes and Jeffrey L. Noebels

Role of Sodium Channels in Epilepsy

David I. Kaplan, Lori L. Isom and Steven Petrou

\section{Mechanisms of Action of Antiseizure Drugs and the Ketogenic Diet Michael A. Rogawski, Wolfgang Löscher and Jong M. Rho}

Epilepsy and Autism

Ashura W. Buckley and Gregory L. Holmes

Immunity and Inflammation in Epilepsy Annamaria Vezzani, Bethan Lang and Eleonora Aronica

Hyperpolarization-Activated Cyclic Nucleotide-Gated (HCN) Channels in Epilepsy Gary P. Brennan, Tallie Z. Baram and Nicholas P. Poolos

The Role of Calcium Channels in Epilepsy Sanjeev Rajakulendran and Michael G. Hanna Interneuron Transplantation as a Treatment for Epilepsy

Robert F. Hunt and Scott C. Baraban

\section{Common Mechanisms Underlying \\ Epileptogenesis and the Comorbidities of \\ Epilepsy \\ Andrey Mazarati and Raman Sankar}

The Diathesis-Epilepsy Model: How Past Events Impact the Development of Epilepsy and Comorbidities

Christophe Bernard

Potassium Channels in Epilepsy

Rüdiger Köhling and Jakob Wolfart

\section{GABAergic Synchronization in Epilepsy Roustem Khazipov \\ Status Epilepticus Syndi Seinfeld, Howard P. Goodkin and Shlomo Shinnar}

Neonatal and Infantile Epilepsy: Acquired and Genetic Models Aristea S. Galanopoulou and Solomon L. Moshé

Epigenetics and Epilepsy

David C. Henshall and Katja Kobow

Microcircuits in Epilepsy: Heterogeneity and Hub

Cells in Network Synchronization Anh Bui, Hannah K. Kim, Mattia Maroso, et al.

For additional articles in this collection, see http://perspectivesinmedicine.cshlp.org/cgi/collection/ 